\title{
Gene expression profiling of microRNAs associated with UCA1 in bladder cancer cells
}

\author{
XIAOJUAN XIE ${ }^{1,2}$, JINGJING PAN $^{1}$, LIQIANG WEI $^{2}$, SHOUZHEN WU $^{3}$, HUILIAN HOU $^{4}, \mathrm{XU} \mathrm{LI}^{3}$ and WEI CHEN ${ }^{1}$ \\ ${ }^{1}$ Department of Clinical Laboratory, The First Affiliated Hospital of Xi'an Jiaotong University, Xi'an, Shaanxi 710061; \\ ${ }^{2}$ Shaanxi Center for Clinical Laboratory, Shaanxi Provincial People's Hospital, Xi'an, Shaanxi 710068; \\ ${ }^{3}$ Center for Translational Medicine, ${ }^{4}$ Department of Pathology, The First Affiliated \\ Hospital of Xi'an Jiaotong University, Xi'an, Shaanxi 710061, P.R. China
}

Received November 11, 2015; Accepted December 27, 2015

DOI: $10.3892 /$ ijo.2016.3357

\begin{abstract}
Emerging evidence indicates that non-coding RNAs, such as lncRNAs and microRNAs, play important roles in diverse diseases, such as cancer, immune diseases and cardiovascular diseases. Interestingly, lncRNAs could directly or indirectly regulate the expression of miRNAs. However, the expression profiling of miRNAs associated with UCA1 in bladder cancer remains unknown. Here, we used Illumina deep sequencing to sequence miRNA libraries from both the UCA1 knockdown and normal high-expression 5637 cells. We identified 225 and 235 miRNAs expressed in 5637 cells of normal high-expression and knockdown of UCA1, respectively. Overall, expression of 75 miRNAs showed significant difference associated with UCA1, of which 38 were upregulated and 37 downregulated with UCA1 knockdown. GO analysis of the host target genes revealed that these aberrantly regulated miRNAs were involved in complex cellular pathways, including biological process, cellular component and molecular function. We selected 8 candidate miRNAs associated with UCA1 and predicted their targeted mRNAs, and found that $\mathrm{p} 27^{\mathrm{kip} 1}$ was a crucial downstream molecule for these 8 miRNAs, especially for miR-196a. KEGG pathway analysis showed that PI3K-Akt signaling pwathway was involved in regulating these 8 candidant miRNAs. Among these 8 candidant miRNAs, we observed the correlation among UCA1, miR-196a and the host target mRNA, p27 ${ }^{\mathrm{kip}}$, in bladder cancer cells and tissues. UCA1 was upregulated by miR-196a
\end{abstract}

Correspondence to: Dr Wei Chen, Department of Clinical Laboratory, The First Affiliated Hospital, School of Medicine, Xi'an Jiaotong University, Xi'an, Shaanxi 710061, P.R. China

E-mail: beibei2010924@163.com

Abbreviations: lncRNA, long noncoding RNA; UCA1, urothelial cancer-associated 1

Key words: urothelial cancer-associated 1, miRNAs, bladder cancer, expression profiling, deep sequencing and positively correlated with miR-196a, whereas UCA1 and miR-196a were negatively correlated with $\mathrm{p} 27^{\mathrm{kip} 1}$, which was downregulated in bladder cancer patients. Thus, our findings provided valuable information on miRNAs associated with UCA1 in bladder cancer, which could be helpful to further explore the related genes and molecular networks fundamental in bladder cancer progression.

\section{Introduction}

Recently, emerging studies have demonstrated that $70-90 \%$ of the human genome is capable of being pervasively transcribed. Surprisingly, $<2 \%$ of the total genome is found to have the ability to encode proteins, indicating that non-coding RNAs account for most of the human transcriptome. In fact, recent evidence has suggested that the human non-coding RNAs include approximately 9,000 small RNAs, 10,000-32,000 long non-coding RNAs (lncRNAs) and 11,000 pseudogenes $(1,2)$. Small non-coding RNAs have been well characterized and categorized by transfer RNAs, microRNAs (miRNAs), smallinterfering RNAs, PIWI-interacting RNAs, small nuclear RNAs, small nucleolar RNAs and transcription initiation RNAs.

lncRNAs can vary in length from 200 nucleotides to 100 kilobases. A previous study demonstrated that lncRNAs play an important role in a diverse range of biological processes (3-5), such as participating in epigenetics, nuclear import, alternative splicing, RNA decay and translation. More importantly, due to their widespread distribution in nucleus, lncRNAs could regulate the transcription of adjacent genes by binding to the specific transcription factors and polymerases $(6,7)$, for example, the expression of microRNAs and their bio-function are mainly regulated by IncRNAs. On the other hand, they can serve as precursors to microRNAs, ceRNA of microRNAs, and natural microRNA sponges. Therefore, aberrant lncRNA expression can cause various human diseases and disorders.

microRNAs, 23-nucleotide-long endogenous RNAs, can bind with their target RNA transcripts by pairing with microRNA response elements (MREs) and then result in degradation or translational repression of the target transcripts $(8,9)$. Recent studies show that microRNAs regulate a number of the 
transcriptions, including the coding and noncoding transcriptome (10). Therefore, a better understanding of microRNA profiling associated with lncRNA in cancer is imperative for the development of novel therapeutic strategies. However, many details remain unknown regarding microRNA profiling regulated by lncRNA.

In this study, we used Illumina deep sequencing to sequence miRNA libraries prepared from the 5637 cells of normal high-expression and knockdown of UCA1, which are specifically upregulated in bladder cancer. Overall, expression of 75 miRNAs showed significant difference associated with UCA1: 38 were upregulated and 37 downregulated. GO analysis of the host target genes revealed that these dysregulated miRNAs are involved in complex cellular pathways, including biological process, cellular component and molecular function. After confirming with RT-qPCR, 8 miRNAs associated with UCA1 were selected to draw the TF-miRNA-mRNA network and enrich KEGG pathway analysis. miR-196a was one of these 8 candidate microRNAs and its targeted gene p2 $7^{\mathrm{kip} 1}$ participated in PI3K-Akt signaling pathway. Then the correlation among UCA1, miR-196a and p2 $7^{\mathrm{kip} 1}$ in bladder cancer cells and patients was analyzed.

\section{Material and methods}

Tissue specimen collection. In this study, 35 bladder cancer tissues and 16 bladder non-tumor tissues were obtained from August 2012 to September 2013 at the Department of Clinical Laboratory, the First Affiliated Hospital of Xi'an Jiaotong University, Xi'an, China. All samples were identified by two independent pathologists. Informed consent was provided by each patient, and this study was approved by the Ethics Committee of the First Affiliated Hospital of Xi'an Jiaotong University and accordance with the Declaration of Helsinki.

Cell culture and treatment. Human bladder cancer cell lines (5637 cell line and UMUC2 cell line) obtained from the American Type Culture Collection (ATCC) were cultured in RPMI-1640 (Gibco, Grand Island, NY, USA) supplemented with $10 \%$ heat inactivated bovine calf serum and $150 \mu \mathrm{g} / \mathrm{ml}$ $\mathrm{G} 418$ at $37^{\circ} \mathrm{C}$ in a humidified atmosphere with $5 \% \mathrm{CO}_{2}$. Stable cell lines with UCA1 knockdown in 5637 cells and UCA1 overexpression in UMUC2 cells were a kind gift of our colleague Zhengkun Li, the Center for Translational Medicine of the First Affiliated Hospital, School of Medicine, Xi'an Jiaotong University (Xi'an, China).

RNA isolation and Illumina sequencing. The total RNA was extracted from UCA1 knockdown 5637 cells and control cells using TRIzol reagent (Invitrogen, Carlsbad, CA, USA) according to the manufacturer's instructions. The RNA concentration was detected by measuring the absorbance at OD $260 \mathrm{~nm}$ (A260) and OD $280 \mathrm{~nm}$ (A280). The purity of RNA was evaluated by the A260/A280 ratio using NanoDrop ND-1000 (NanoDrop Technologies, Wilmington, DE, USA). The integrity of RNA samples were further measured by $28 \mathrm{~S} / 18 \mathrm{~S}$ ratio using Agilent 2100 Bioanalyzer (Agilent Technologies, Santa Clara, CA, USA) and all RNA samples were verified as intact with the RNA integrity number $(\mathrm{RIN})>7$. The two RNA samples were then sent to
Huada BGI-Tech (Shanghai, China) for Illumina sequencing of the small RNAs.

Analysis of sequencing data. The small RNA sequencing reads obtained were then subjected to the following: i) reads were selected by removing the low-quality reads with the Solexa Chastity quality filter; ii) the adapter sequences were trimmed and the reads longer than 15 were retained; and iii) low two-copy sequences were discarded. The filtered short reads were then mapped to the Repbase database (available online: http://www. girinst.org/repbase/), Rfam database, NCBI database (available online: http://www.ncbi.nlm.nih.gov/) (available online: http:// rfam.janelia.org/). The unmappable sequences not mapped to miRBase 19.0 were used to predict potentially novel candidate miRNAs with the Mfold RNA folding prediction web server (available online: http://mfold.rna.albany.edu/).

Identification of differentially-expressed miRNAs. To compare the differential miRNA expression in 5637 cells of normal high-expression and knockdown of UCA1, the number of raw tags in each library was normalized to tags per million of the total miRNA reads. Differentially-expressed miRNAs were determined according to the criterion of a fold change of $\geq 2$ in the sequence counts between libraries.

Real-time PCR. The total RNA was extracted from cells or bladder cancer patient tissues using TRIzol reagent (Invitrogen) as described above. RNA was subjected to reverse transcription reactions using the PrimeScript RT reagent kit (Invitrogen). Selected miRNAs were subjected to RT-qPCR using SYBR Premis Ex Tag II (Takara, Dalian, China) and monitored with the CFX96 Touch Real-time PCR detection system (BioRad, Hercules, CA, USA). The PCR reaction mixture $(20 \mu \mathrm{l})$ consisted of $2 \mu \mathrm{l}$ of cDNA, $10 \mu \mathrm{l}$ of $2 \mathrm{X}$ SYBR-Green PCR master mix, $0.8 \mu \mathrm{l}$ each of the microRNA-specific forward primer, a universal reverse primer $(10 \mu \mathrm{M})$ and $6.4 \mu \mathrm{l}$ of nuclease-free water. The cycling parameters were $94^{\circ} \mathrm{C}$ for $5 \mathrm{~min}$ followed by 40 cycles of $94^{\circ} \mathrm{C}$ for $30 \mathrm{sec}$ and $60^{\circ} \mathrm{C}$ for 30 sec. U6 was used as the endogenous control for normalization. The relative expression levels were calculated with the $2^{-\Delta \mathrm{Ct}}$ method. All the miRNA primers were obtained from Ribobio (Guangzhou, China). Three independent biological replicates were performed.

miRNA target prediction. The potential target genes of the differentially-expressed miRNAs were predicted with four miRNA target prediction algorithms, miRanda (available online: http://www.microrna.org/), miRDB (available online: http://www.mirdb.org/), RNAhybrid (available online: http://bibiserv.techfak.uni-bielefeld.de/) and TargetScan (available online: http://www.targetscan.org/).

Gene ontology and KEGG pathway analysis. The Gene Ontology program (available online: http://www.gene ontology.org) was used for GO annotation and enrichment analysis of the miRNA target genes based on: cell component, biological process and molecular function. The false discovery rate (FDR) was evaluated using the default parameters to adjust the P-values. Genes with FDR $\leq 0.05$ were considered to be significantly enriched in the target candidate genes. Enriched 
Table I. The summary of data produced by small RNA sequencing.

\begin{tabular}{lrrrr}
\hline & \multicolumn{2}{c}{$\mathrm{N}$} & \multicolumn{2}{c}{$\mathrm{S}$} \\
\cline { 2 - 4 } Type & \multicolumn{1}{c}{ Count } & Percentage & Count & Percentage \\
\hline Total reads & $17,747,882$ & - & $18,522,937$ & 100.00 \\
High quality & $11,932,390$ & 100.00 & $11,941,633$ & 0.04 \\
3' adapter null & 7,725 & 0.06 & 5,284 & 0.09 \\
Insert null & 14,454 & 0.12 & 30,770 & 3.28 \\
5' adapter contaminants & 409,756 & 3.43 & 391,361 & 0.57 \\
Smaller than 18 nt & 47,127 & 0.39 & 67,854 & 0.04 \\
PolyA & 6,368 & 0.05 & 4,329 & 95.98 \\
Clean reads & $11,446,960$ & 95.93 & $11,462,035$ & \\
\hline
\end{tabular}

$\mathrm{N}$, sample of UCA1 normal high-expression; S, sample of UCA1 knockdown.

KEGG pathway analyses of 8 candidate miRNA-targeted genes were performed by bioinformatics tool DAVID 6.7. $\mathrm{P}$-value of significance by Fisher's test was set at $\mathrm{P}<0.05$.

Western blot analysis. The 5637 or UMUC2 cells were pelleted and then lysed by RIPA buffer (Thermo Scientific, Waltham, MA, USA) containing protease inhibitors (Roche). After SDS-PAGE resolution and membrane transfer, the target proteins were probed with primary antibody against human p2 $7^{\mathrm{kip} 1}$ or $\beta$-actin (Abcam, Cambridge, UK) followed by incubation with HRP-conjugated secondary antibodies. Then, the bands were visualized using a chemiluminescence detection kit (Pierce, Rockford, IL, USA) and the specific bands were recorded on X-ray film.

Immunohistochemistry (IHC). Tissue sections were deparaffinized and rehydrated. Endogenous peroxidase activity was blocked with $0.3 \%$ hydrogen peroxide for $20 \mathrm{~min}$. For antigen retrieval, slides were microwave treated and boiled in a $10 \mathrm{mM}$ citrate buffer ( $\mathrm{pH}$ 6.0) for $10 \mathrm{~min}$. Nonspecific binding was blocked with $10 \%$ normal rabbit serum for $20 \mathrm{~min}$. The slides were incubated with monoclonal mouse anti-human p27 $7^{\mathrm{kip} 1}$ (1:150 dilution; Abcam) overnight at $4^{\circ} \mathrm{C}$ in a moist chamber. The slides were sequentially incubated with biotinylated rabbit anti-mouse immunoglobulin at a concentration of 1:150 for $30 \mathrm{~min}$ at $37^{\circ} \mathrm{C}$ and then reacted with a streptavidin-peroxdase conjugate for $30 \mathrm{~min}$ at $37^{\circ} \mathrm{C}$ and diaminobenzidine as a chromogen substrate. The nucleus was counterstained using Meyer's hematoxylin.

Statistical analysis. Statistical analysis was performed with SPSS software (SPSS standard version 11.5; SPSS Inc., Chicago, IL, USA). The association analysis was assessed by the Chi-square test. Data are represented as mean \pm SD. $\mathrm{P}$-value of $<0.05$ was considered significant.

\section{Results}

Analysis of miRNAs from deep sequencing. We sequenced two small RNA libraries built from 5637 cells of normal high-expression and knockdown of UCA1, which contained
$17,747,882$ and $18,522,937$ raw reads, respectively. In 5637 cells, $82.40 \%$ of total reads and $48.06 \%$ of unique reads could be mapped to the human reference genome, and in 5637 cells of UCA1 knockdown, the proportions were 85.46 and $38.49 \%$, respectively. After quality control procedures, 11,446,960 and 11,462,035 short reads were kept for further analyses for 5637 cells of normal high-expression or knockdown of UCA1, respectively (Table I). We found that 12.68 and $13.15 \%$ of the clean reads correspond to miRNAs for 5637 cells of normal high-expression or knockdown of UCA1, respectively. The remaining clean reads could be mapped to other genomic locations, corresponding to other different kinds of small RNAs, including repeats, snRNA, snoRNA, rRNA and tRNA. The chromosomal distributions of these sRNAs from 5637 cells of normal high-expression or knockdown of UCA1 are shown in Fig. 1A and B, respectively. Most of the small RNAs (91.30 and $89.66 \%$, respectively) from both libraries were $21-24 \mathrm{nt}$ in length (Fig. 1C and D). Importantly, 22-nt RNAs were the most abundant, accounting for 65.29 and $62.26 \%$ of the small RNAs in 5637 cells of normal high-expression or knockdown of UCA1, respectively, which is consistent with the typical size of miRNA from Dicer-derived products. Analysis of the first nucleotide bias in these miRNAs is presented in Fig. 1E and F. The results showed that uridine (U) dominated the first position, whereas guanine $(G)$ was the least favored first base, which is a characteristic feature of miRNAs. Furthermore, Fig. $1 \mathrm{G}$ and $\mathrm{H}$ show the analysis of the each nucleotide bias in these miRNAs, suggesting that uridine (U) had higher frequence, especially in position 1, 6, 9, 13, 14, 16, 18, 21 and 22.

Analysis of miRNAs associated with UCA1. Of the 829 human mature miRNAs deposited in miRBase were identified in our two libraries (235 in UCA1 normal high-expression cells and 225 in UCA1 knockdown cells). Among these, 75 miRNAs were found in both libraries and significantly differentially expressed by $\geq 2$-fold from 5637 cells of normal high-expression or knockdown of UCA1 $(\mathrm{P}<0.05)$, in which 38 miRNAs were highly expressed (upregulated), and 37 miRNAs had low expression (downregulated) in UCA1 knockdown cells (Fig. 2A and B), suggesting that these miRNAs are 

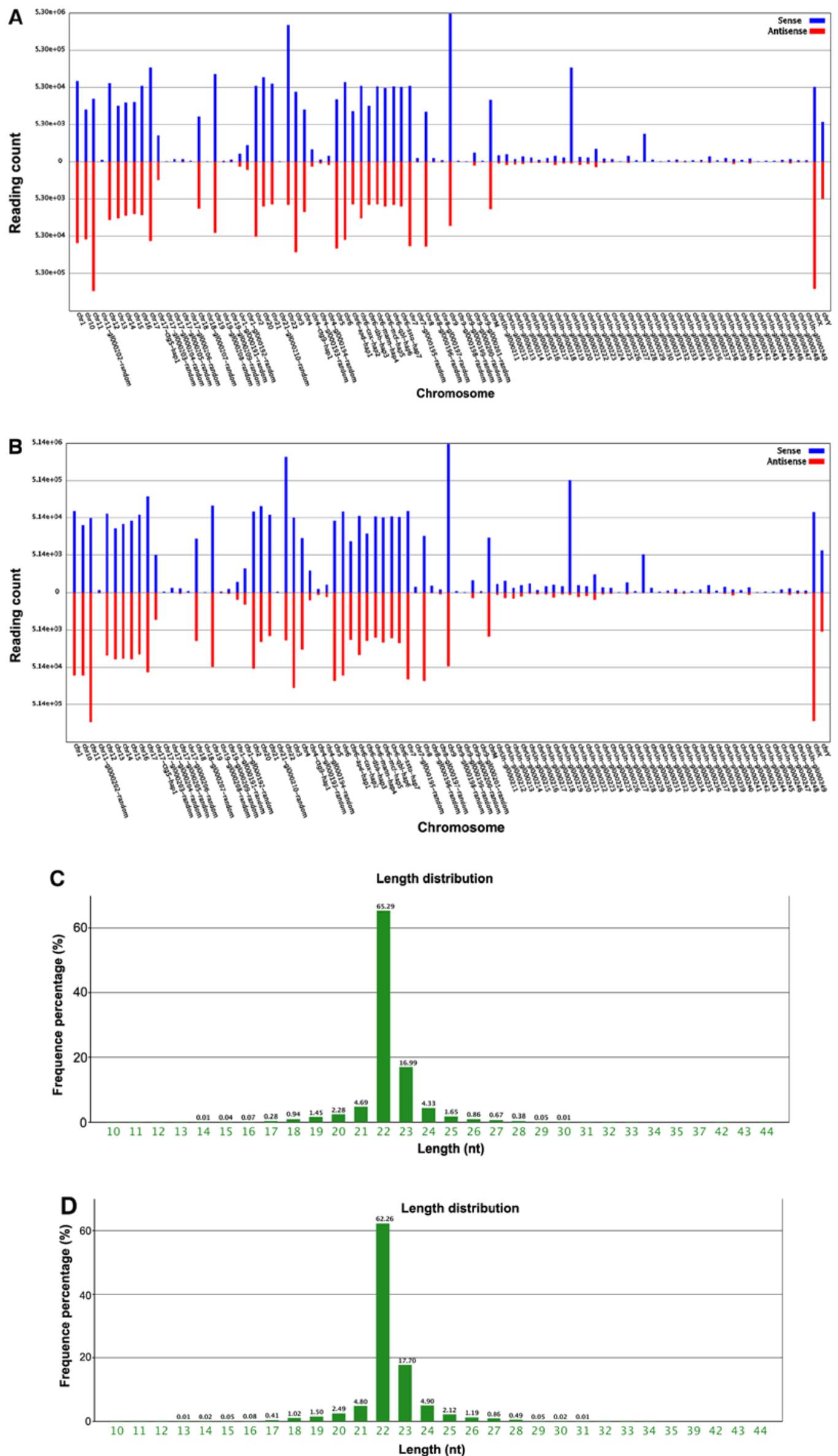

Figure 1. The characterization of small RNA from deep sequencing. The chromosomal distribution of sRNAs by deep sequencing from 5637 cells of UCA1 normal high-expression (A) and UCA1 knockdown (B). Length distribution of small RNA read sequences were analyzed in 5637 cells of UCA1 normal high-expression (C) and UCA1 knockdown (D). 

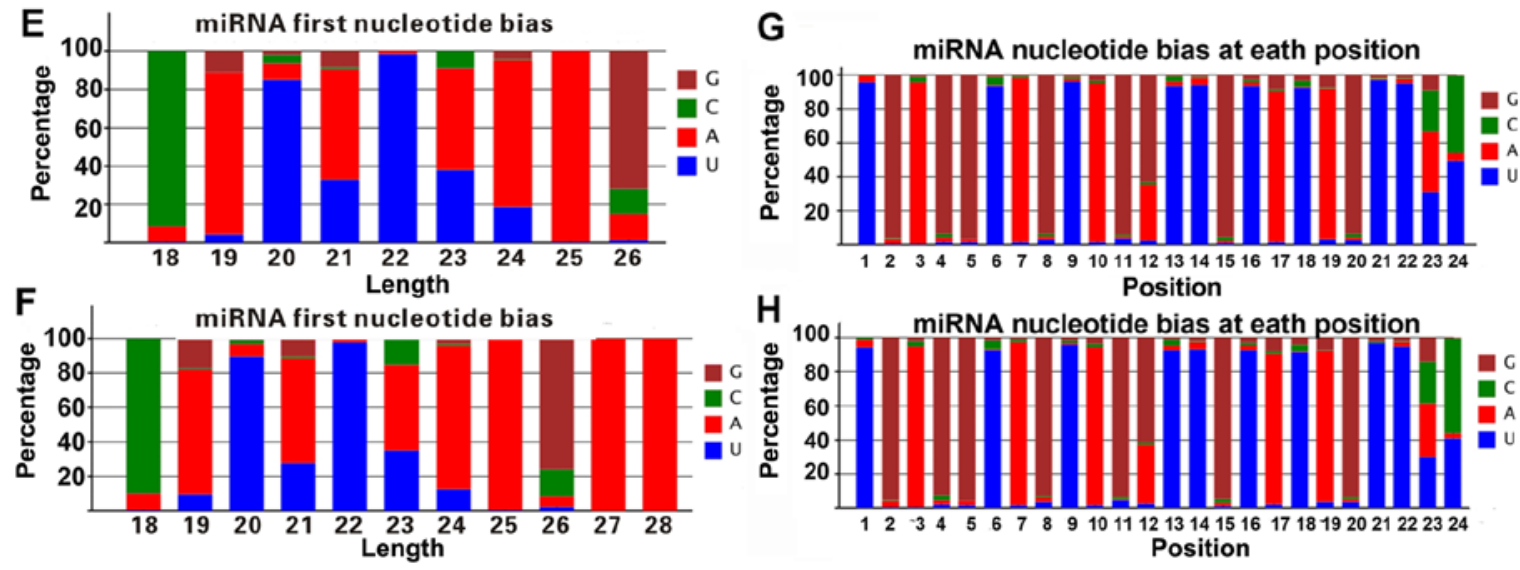

Figure 1. Continued. The first nucleotide bias of UCA1 normal high-expression (E) and UCA1 knockdown (F) were analyzed. Each nucleotide bias of UCA1 normal high-expression $(\mathrm{G})$ and UCA1 knockdown $(\mathrm{H})$ was analyzed.
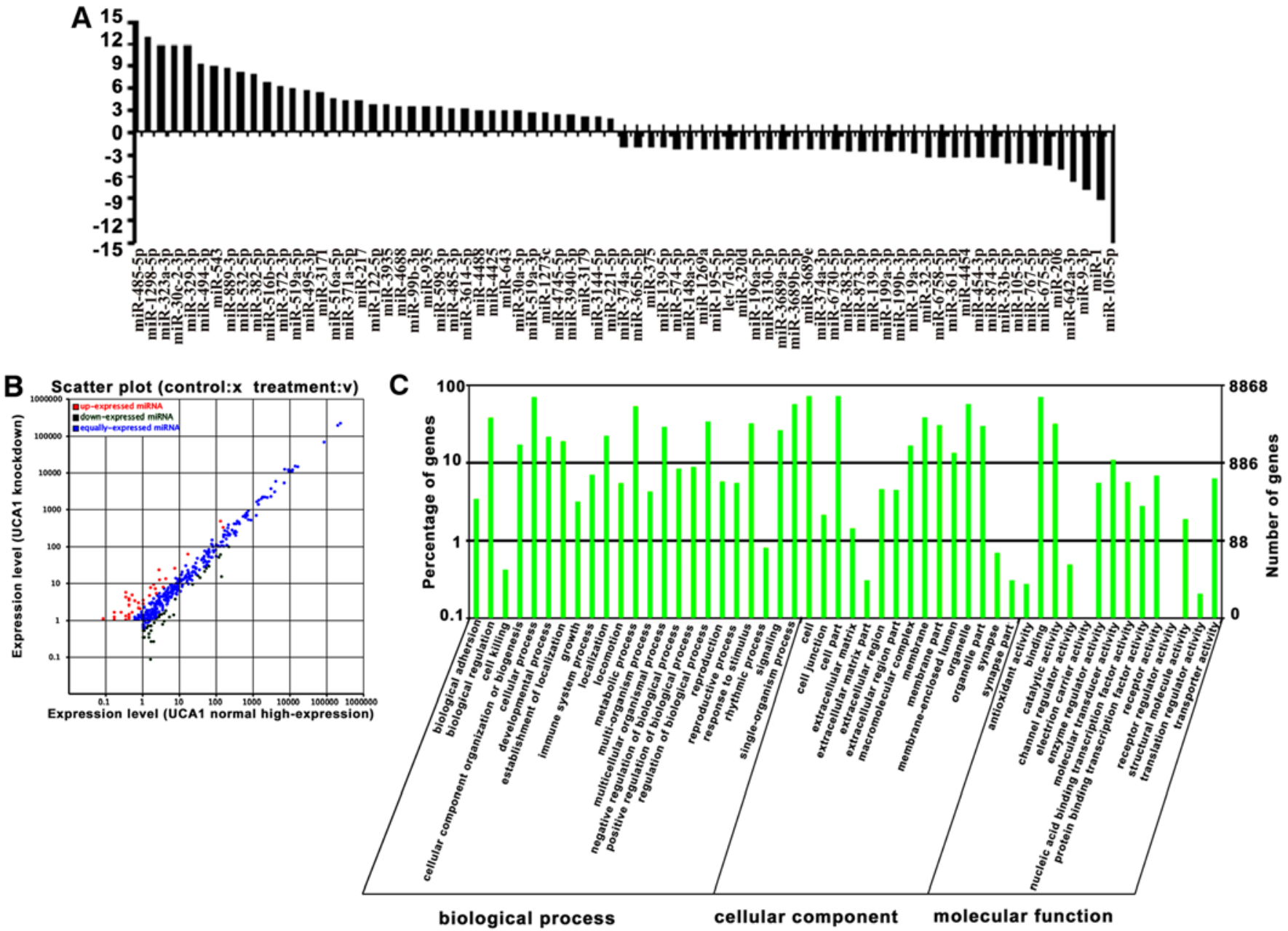

Figure 2. Differentially expressed miRNAs associated with UCA1. (A) Expression of 75 miRNAs showed significant difference associated with UCA1: 38 were upregulated and 37 downregulated after UCA1 knockdown in 5637 cells. y-axis indicates the fold-change of miRNA expression difference between 5637 cells of UCA1 normal high-expression and UCA1 knockdown. (B) Scatter plot analysis of the 75 different miRNAs in (A). (C) The GO annotation showed the genes regulated by the differentially expressed miRNAs associated with UCA1 in biological process, cellular component and molecular function.

associated with UCA1 expression in bladder cancer cells. To further understand the physiological functions of the miRNAs associated with UCA1, the potential targets of the up- and downregulated miRNAs were predicted with four miRNA target prediction software programs: TargetScan, miRanda, Clip-Seq and miRDB. We found tens of thousands of putative targets in the combined outputs of the programs. All of the predicted target genes of miRNAs were then subjected sepa- 

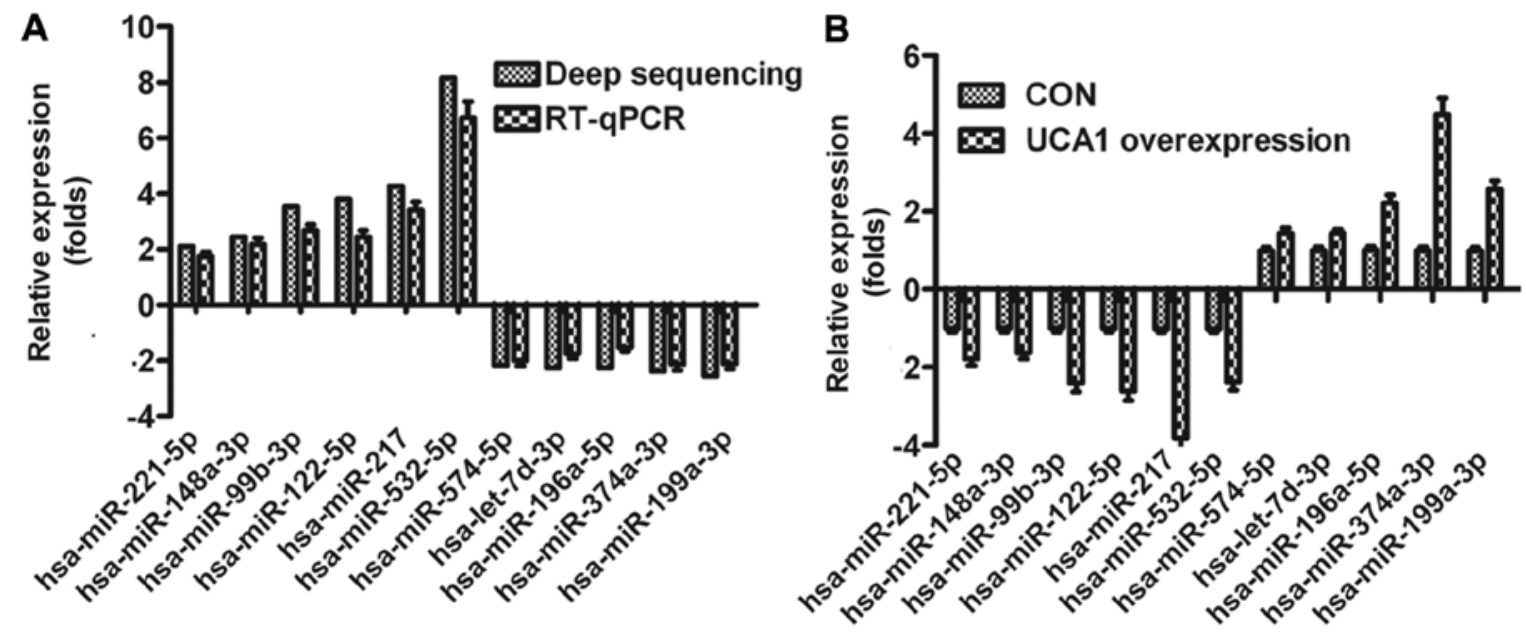

Figure 3. qRT-PCR validation of miRNAs identified by deep sequencing. According to the literatures and the expression difference, 11 miRNAs were selected and detected by real-time PCR in 5637 cells of UCA1 normal high-expression and UCA1 knockdown. The multiple of expression differences of these 11 miRNAs determined with deep sequencing and RT-qPCR were compared (A). The expression levels of these 11 miRNAs were observed in UMUC2 cells transfected by UCA1 (B). qRT-PCR was repeated by 3 times.

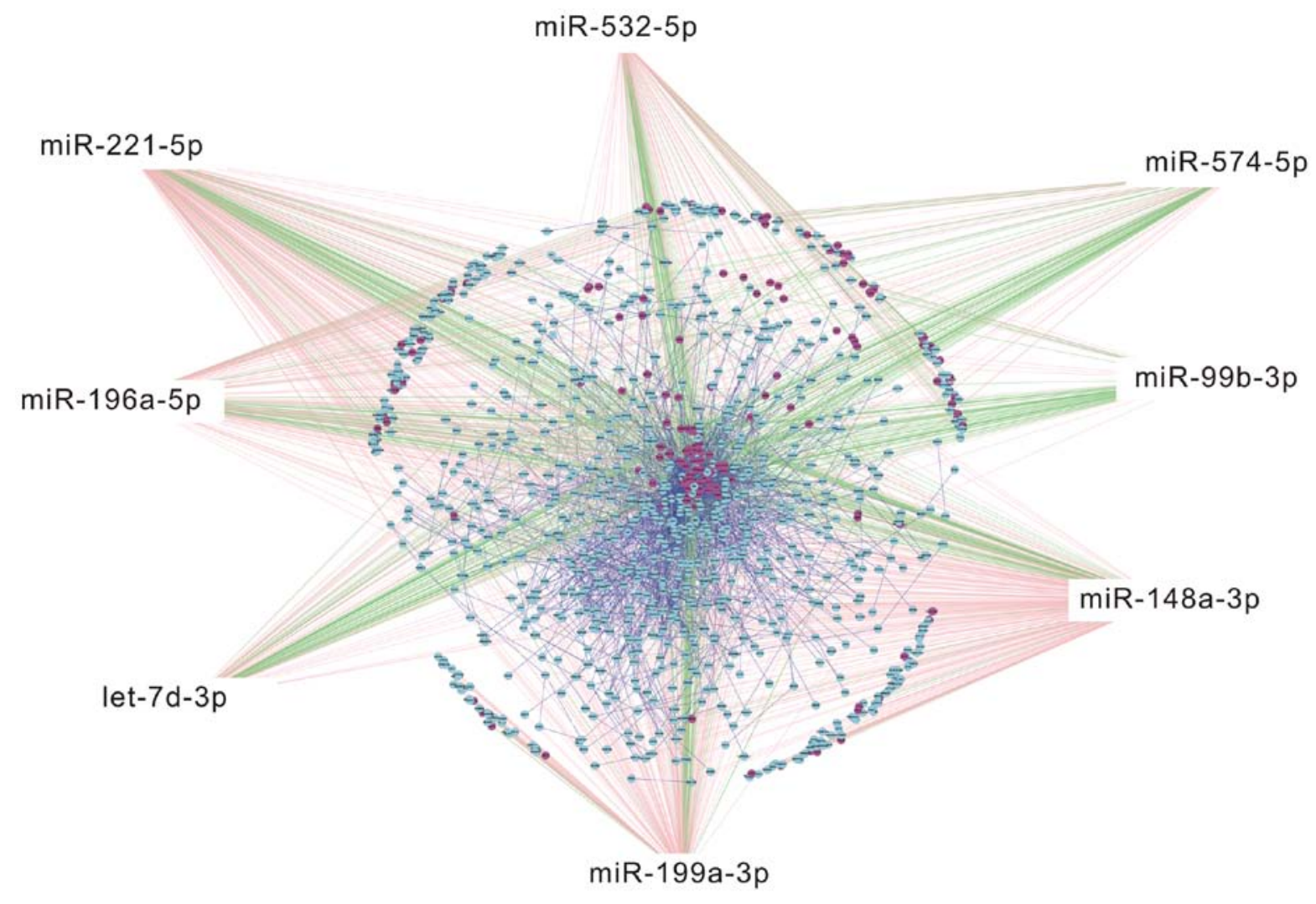

Figure 4. TF-miRNA-mRNA network by bioinformatics analysis. Eight candidate miRNAs were selected to draw TF-miRNA-mRNA network.

rately to GO analysis. The GO category analysis revealed that these genes fell into three major categories: biological process, cellular component and molecular function (Fig. 2C).

Validation of deep sequencing results by $R T-q P C R$. To validate the results obtained from deep sequencing, we selected 11 miRNAs already known in miRBase associated with bladder cancer in this study for validation by qRT-PCR, respectively. The expression levels of the miRNAs determined with deep sequencing and RT-qPCR were compared (Fig. 3A). On the whole, the relative expression levels of the selected miRNAs detected with RT-qPCR were lower than the relative expression levels determined with deep sequencing, which may result from the difference in the sensitivity of the two methods. To further confirm that the sequencing miRNAs were associated with UCA1 in bladder cancer, we detected the expression of the above 11 miRNAs in UMUC2 cells transfected by UCA1. As shown in Fig. 3B, expression of 11 miRNAs was positively correlated to UCA1, which also validated the sequencing results. 
A

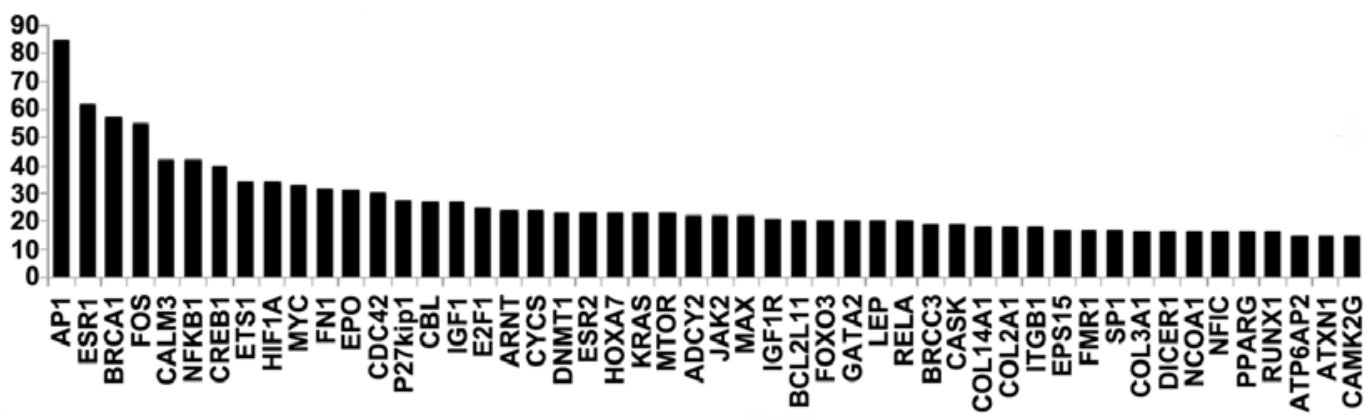

B

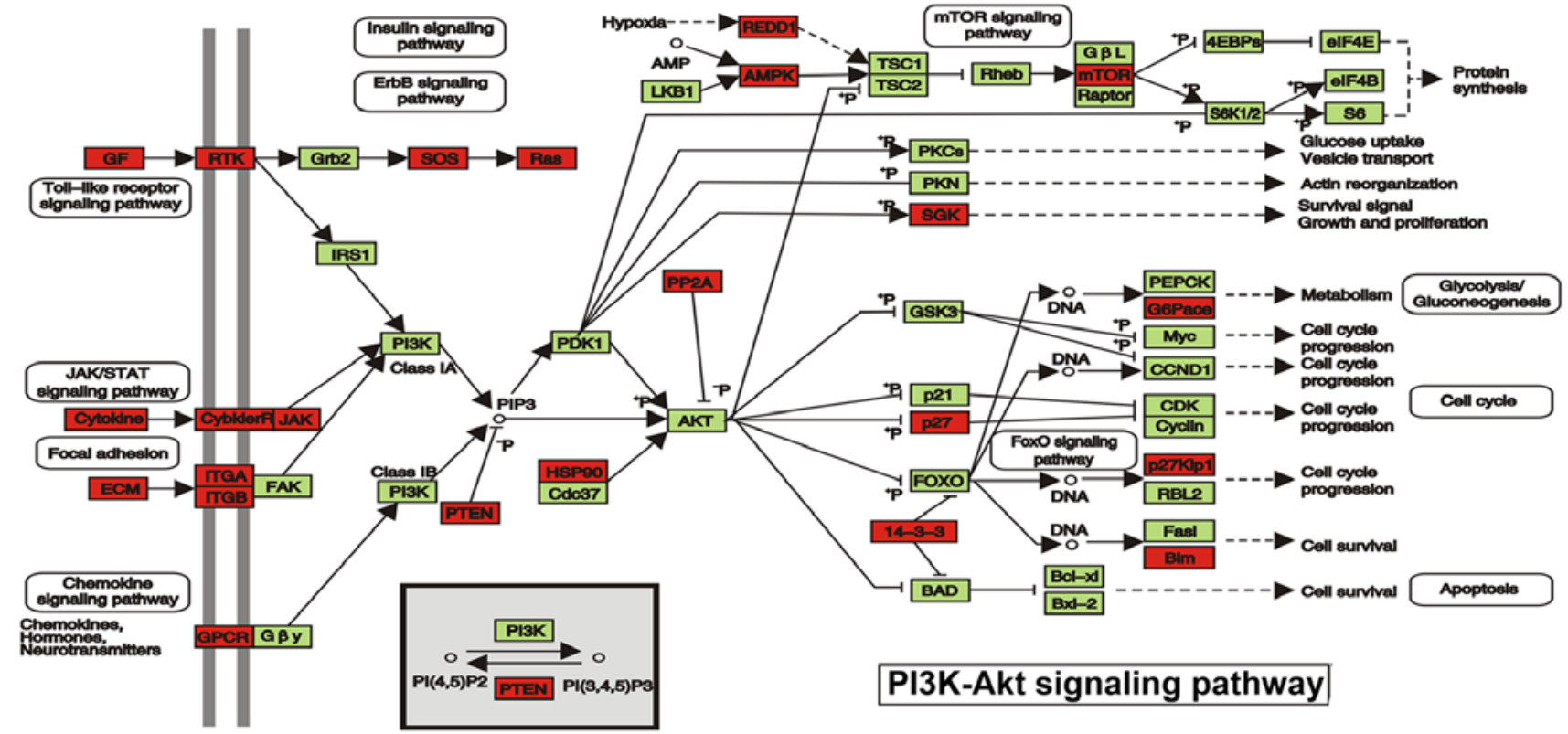

Figure 5. Important genes and signal pathway by bioinformatics analysis. (A) The 50 genes and transcription factors in TF-miRNA-mRNA network are listed in order of importance. (B) KEGG pathway analysis showed that PI3K-Akt signaling was responsible for the bio-function of the 8 candidate miRNAs. Red target genes of miRNAs by predition. Green-genes of PI3K-Akt signaling pathway.

Bioinformatics analysis of 8 candidate miRNAs. Eight miRNAs associated with UCA1 selected from the above 11 miRNAs were selected to draw TF-miRNA-mRNA network (Fig. 4). The top 50 genes and transcription factors in TF-miRNA-mRNA network are listed based on the relevance (Fig. 5A). KEGG pathway analysis was employed to predict target genes of the 8 miRNAs and six signaling pathways were found statistically significant $(\mathrm{P}<0.05)$ (Table II). p2 $7^{\mathrm{kip} 1}$ was found as a crucial target gene for the 8 candidate miRNAs. In addition, enriched KEGG pathway analysis showed that the target gene $\mathrm{p} 27^{\mathrm{kip} 1}$ regulated by one of the 8 candidate miRNAs, miR-196a, was involved in PI3K-Akt signaling pathway (Fig. 5B), suggesting that PI3K-Akt signaling played an important role in the regulation of the 8 candidate miRNAs.

The correlation among UCA1, miR-196a and p27kipl in bladder cancer cells and patients. Next, we observed miR-196a expression in bladder cancer and focused on the regulation of UCA1 on miR-196a. The result revealed that miR-196a was significantly decreased in 5637 cells after transfection by siUCA1 (Fig. 6A and B), whereas the levels of p27 $7^{\mathrm{kip} 1}$ was remarkably increased (Fig. 6C and D). In contrast, overexpression of UCA1 could significantly upregulate miR-196a in UMUC2 cells (Fig. 6E and F), whereas p27 $7^{\mathrm{kip} 1}$ was downregulated in UMUC2 cells of ectopic UCA1 expression (Fig. 6G and H). Besides, the expression of UCA1 and miR-196a were statistically significantly higher in bladder cancer tissues than those in non-tumor bladder tissues (Fig. 7A and B), whereas p27 ${ }^{\text {kip1 }}$ was downregulated in bladder cancer tissues compared with non-tumor bladder tissues (Fig. 7C). Furthermore, p27 ${ }^{\text {kip1 }}$ expression was also detected in bladder cancer tissues and non-tumor bladder tissues using IHC. As shown in Fig. 7D, $\mathrm{p} 27^{\mathrm{kip} 1}$ staining was remarkably decreased in bladder cancer tissues than that in non-tumor bladder tissues. Whether UCA1 expression was correlated with miR-196a and p27 ${ }^{\text {kipl }}$ in bladder cancer was investigated as well. As expected, UCA1 was positively correlated with miR-196a in bladder cancer patients (Fig. 7E), whereas the levels of UCA1 and miR-196a were negatively correlated with $\mathrm{p} 27^{\mathrm{kip} 1}$ in bladder cancer, respectively (Fig. 7F and G). These data suggested that miR-196a was regulated by UCA1 in bladder cancer and implemented its bio-function via inhibiting $\mathrm{p} 27^{\mathrm{kip} 1}$ expression, which was also confirmed by the valuable information of miRNA profiling associated with UCA1 in bladder cancer. 
Table II. KEGG pathway analysis of 8 candidate miRNAs.

\begin{tabular}{|c|c|c|c|c|c|}
\hline Pathway & Id & $\begin{array}{l}\text { Sample } \\
\text { no. }\end{array}$ & $\begin{array}{l}\text { Background } \\
\text { no. }\end{array}$ & P-value & Genes \\
\hline $\begin{array}{l}\text { Proteoglycans } \\
\text { in cancer - } \\
\text { Homo sapiens } \\
\text { (human) }\end{array}$ & hsa05205 & 31 & 225 & 8.59E-06 & $\begin{array}{l}\text { MTORISMAD2INRASISDC4IITGA5IPAK1ISDC2|ESR1| } \\
\text { FZD6IPXNIPRKACBIIGF1ICAMK2GIPPP1CBIWNT1| } \\
\text { FRS2IIGF1RIHPSE2|CDC42|WNT10BIMRASIROCK1| } \\
\text { CBLBIITGB1|FN1IPDCD4|SOS2|CBLI } \\
\text { KRASIERBB3|ERBB4| }\end{array}$ \\
\hline $\begin{array}{l}\text { ErbB signaling } \\
\text { pathway }\end{array}$ & hsa04012 & 14 & 88 & 0.000581 & $\begin{array}{l}\text { CBLB|CDKN1B|PAK4|NRASISOS2|MTORICBLICAMK2G| } \\
\text { KRASIPAK1ITGFAIPAK7|ERBB3|ERBB4| }\end{array}$ \\
\hline $\begin{array}{l}\text { ECM-receptor } \\
\text { interaction }\end{array}$ & hsa04512 & 14 & 88 & 0.000581 & $\begin{array}{l}\text { ITGA11|THBS2|ITGB1|COL3A1|CD47|COL4A1|COL2A1| } \\
\text { COL4A5IITGB8ISDC4|ITGA5|SDC2IITGA3|FN1| }\end{array}$ \\
\hline Glioma & hsa05214 & 11 & 65 & 0.001305 & $\begin{array}{l}\text { PTENINRASIIGF1RISOS2|IGF1|CAMK2G|RB1|CALM3| } \\
\text { KRASIMTORITGFAI }\end{array}$ \\
\hline $\begin{array}{l}\text { PI3K-Akt } \\
\text { signaling } \\
\text { pathway - } \\
\text { Homo sapiens } \\
\text { (human) }\end{array}$ & hsa04151 & 34 & 346 & 0.002319 & 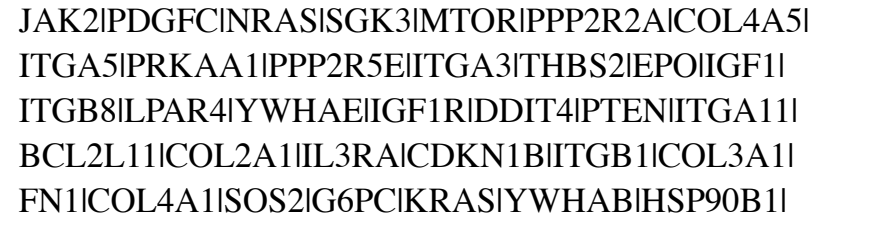 \\
\hline Focal adhesion & hsa04510 & 23 & 206 & 0.002327 & $\begin{array}{l}\text { PAK4|PDGFCIITGA5|PAK1|PPP1CB|ITGA3|THBS2|PXN| } \\
\text { IGF1IITGB8IPAK7IIGF1R|CDC42|ITGA11|ROCK1|COL2A1| } \\
\text { COL4A5|ITGB1|COL3A1|FN1|COL4A1|SOS2|PTEN| }\end{array}$ \\
\hline
\end{tabular}
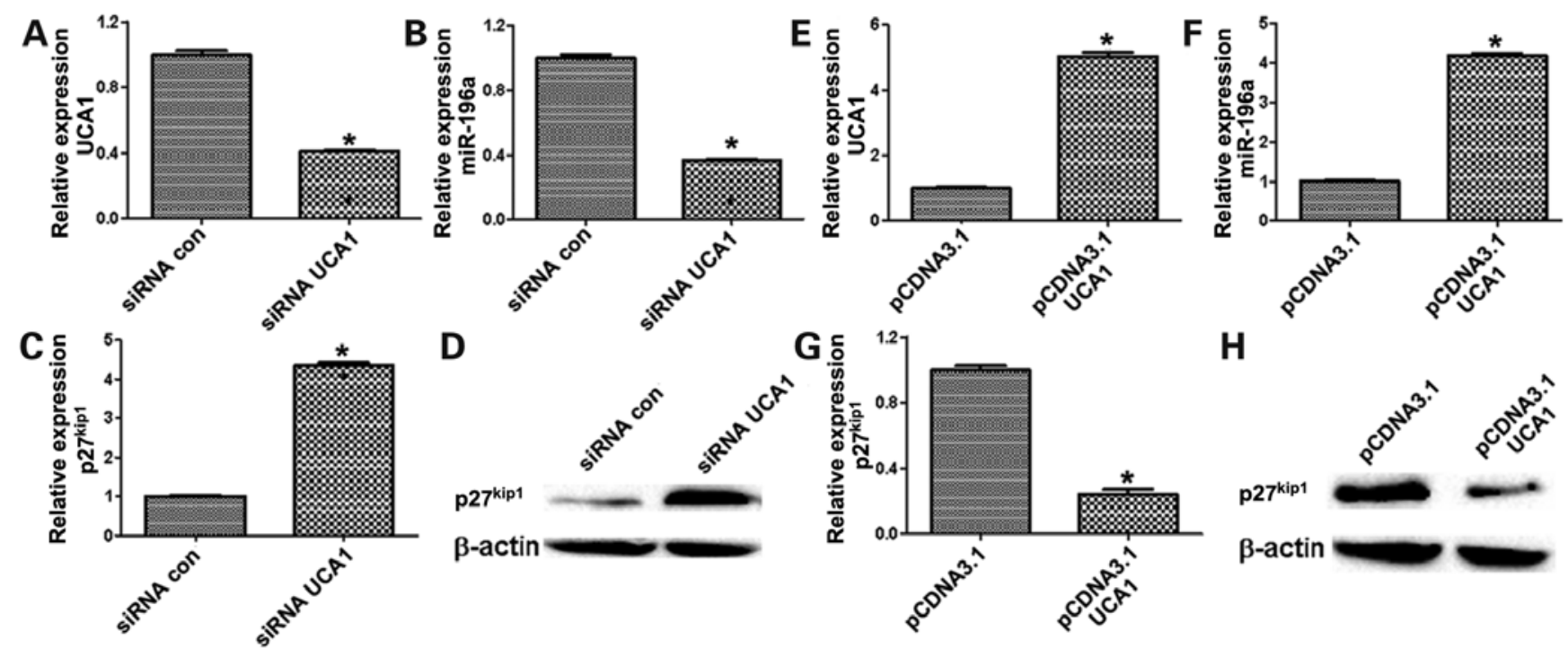

H

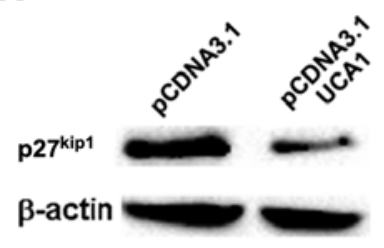

Figure 6. The expression and correlation analysis among UCA1, miR-196a and p27 kipl in bladder cancer cells. 5637 cells were transfected by siUCA1. The levels of UCA1 (A), miR-196a (B) and p27 $7^{\mathrm{kipl}}$ (C) were detected by real-time PCR, respectively. The level of p27 $7^{\mathrm{kipl}}$ protein was analyzed using western blot analysis (D). pCDNA3.1-UCA1 was transfected into UMUC2 cells. The expression of UCA1 (E), miR-196a (F) and p27 $7^{\mathrm{kipl}}(\mathrm{G})$ were analyzed by real-time PCR respectively. p2 $7^{\text {kipl }}$ protein level was detected via western blot analysis (H). qRT-PCR was repeated 3 times.

\section{Discussion}

Diseases, especially cancer, are often associated with the dysregulation of gene and aberrant transcriptomes, which includes the production of abnormal levels of protein-coding mRNAs and deregulated expression of the noncoding dimension of the human genome. This is especially surprising since recent findings of the human transcriptome have revealed that over three quarters of the genome is transcribed $(2,11)$, including mRNA, small non-coding RNA and lncRNAs. The non-coding transcriptome and their transcriptions play an important role in determining the greater complexity of higher eukaryotes and in disease pathogenesis (12-14). Especially in cancer, the noncoding transcriptome is often dysregulated (15). 

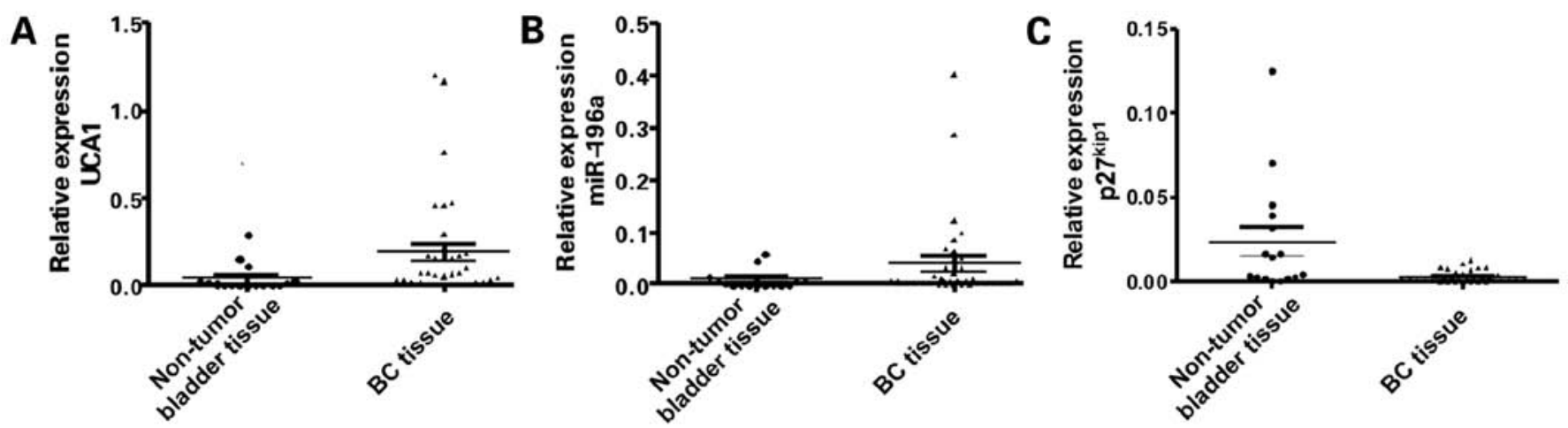

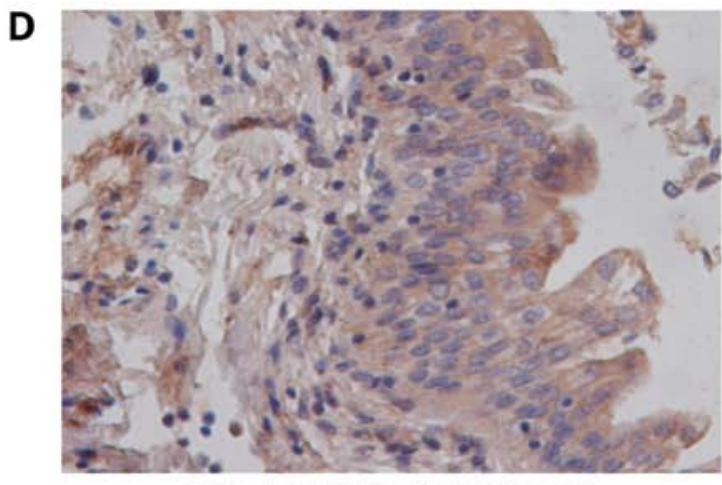

Non-tumor bladder tissue

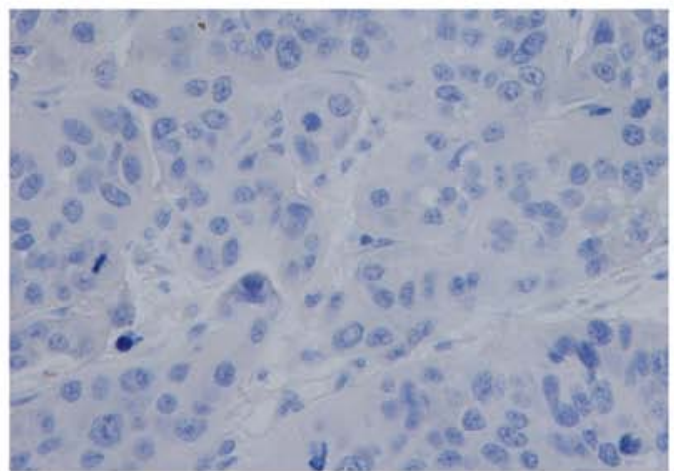

Bladder cancer tissue
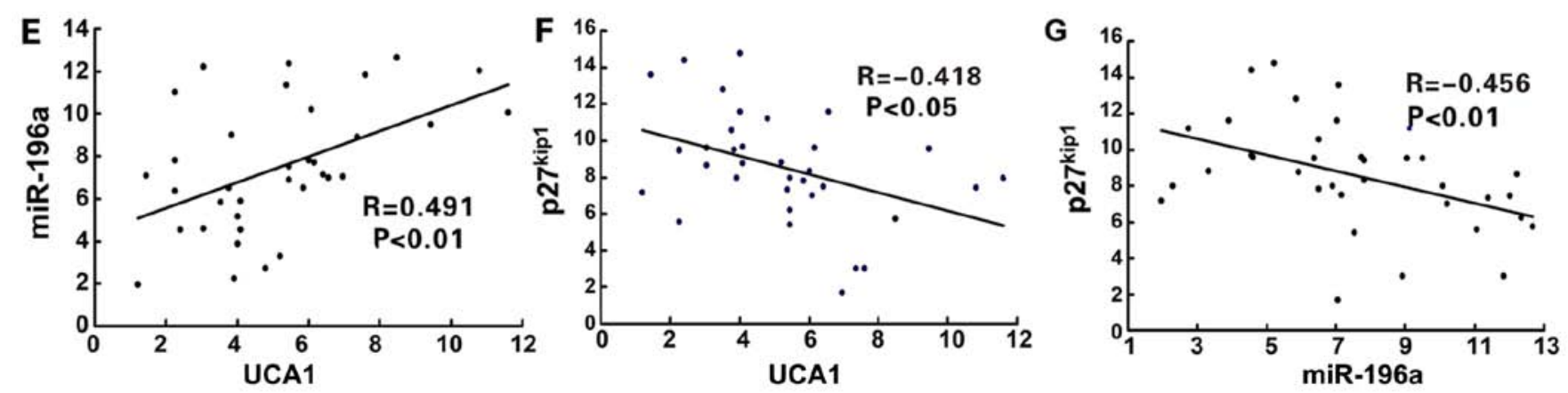

Figure 7. The expression and correlation analysis among UCA1, miR-196a and p27 ${ }^{\mathrm{kip} 1}$ in bladder cancer tissues. The expression levels of UCA1 (A), miR-196a (B) and $\mathrm{p} 27^{\mathrm{kip} 1}(\mathrm{C})$ were detected by real-time PCR in 35 bladder cancer tissues, and 16 nontumor bladder tissues. p27 $7^{\mathrm{kip} 1}$ staining was observed in these tissues. The representative staining of p27 $7^{\mathrm{kipl}}$ is showed (D), $\mathrm{x} 400$. UCA1 was positively related to miR-196a (E) and negatively to p27 $7^{\mathrm{kip} 1}$ (F) in bladder cancer pateint. miR-196a was negtively correlated with $2^{\text {kipl }}$ in bladder cancer $(\mathrm{G})$. qRT-PCR was repeated 3 times.

Over the past decade, many studies focused on the microRNAs in cancer and have demonstrated that microRNAs are involved in cancer biology through controlling expression of their target mRNAs. Thus, microRNAs play an important role in facilitating tumor growth, invasion, angiogenesis, and even immune evasion $(16,17)$. Additionally, tumor microRNA profiles in cancer are explored and the different expression of microRNAs in tumor is related with subtypes, patient survival and treatment response (18-20). Importantly, cancer-associated microRNA biomarkers can be detected in biological fluids, allowing less-invasive monitoring (21).

Accumulating evidence suggests that lncRNAs play an important role in tumorigenesis (22) and in the regulation at chromatin organization, at transcriptional, and post-transcriptional levels (23). LncRNAs have been considered as essential regulators in diverse aspects of biology. LncRNAs can serve as important players in post-transcriptional regulation, such as mRNA editors, mRNA splicing regulators, and reservoir of small ncRNAs. For example, lncRNAs can regulate miRNAs by competing endogenous RNAs (ceRNAs) or natural microRNA sponges (14). Considering the roles of lncRNAs and microRNAs in cancer, a better understanding of microRNA profiling associated with lncRNA in cancer is imperative for the development of novel therapeutic strategies.

Our previous studies demonstrated that IncRNA and UCA1 played an important role in cell proliferation, cell cycle, invasion and metastasis in bladder cancer $(24,25)$. However, it remains unknown whether microRNAs participate in these processes regulated by UCA1. Deep sequencing is considered as a powerful strategy for identifying novel miRNAs and studying 
the expression profiles of miRNA in different species at various developmental stages and in diseased states $(26,27)$. In the present investigation, we analyzed the microRNA profiling associated with UCA1 in bladder cancer by deep sequencing.

The miRNAs frequently varied from their miRBase reference sequences, displaying multiple mature isoforms. Thus, we compared all of these isoforms in our libraries, finding that $3^{\prime}$ heterogeneity was more common than $5^{\prime}$ heterogeneity in the miRNAs from deep sequencing. This is consistent with the findings of other miRNA deep sequencing studies and the observation that most variability occurs in either the Dicer1 or Drosha cleavage position within the pre-miRNA hairpin (28-30). We further analyzed the first nucleotide bias and the each nucleotide bias in the miRNAs from sequencing. Uridine (U) dominated the first position and the each nucleotide bias, whereas guanine $(\mathrm{G})$ was the least favored in this study, which is a characteristic feature of miRNAs.

According to the deep sequencing results, we found expression of 75 miRNAs significantly associated with UCA1: 38 were upregulated and 37 downregulated. To explore the potential physiological functions of the miRNAs associated with UCA1, we analyzed the potential targets of the up- and downregulated miRNAs with four miRNA target prediction software programs, and performed Gene ontology analysis. GO analysis revealed that 75 significantly differentially expressed miRNAs participated in a variety of biological processes potentially important to bladder cancer progression, including biological process, cellular component and molecular function.

To confirm the sequencing data, 11 candidate miRNAs were randomly selected and detected by real-time PCR. On the whole, the relative expression levels of the selected miRNAs detected with RT-qPCR were lower than the relative expression levels determined with deep sequencing. The difference may be due to the difference in the sensitivity of the methods.

In addition, we constructed a network regulated by 8 candidate miRNAs selected from the above 11 miRNAs, predicted their transcription factors and targeting mRNAs. In TF-miRNA-mRNA network, p27 ${ }^{\text {kipl }}$ was a crucial gene. Kyoto Encyclopedia of Genes and Genomes (KEGG) pathway analysis found all these 8 candidate miRNAs participate in PI3K-Akt signaling, suggesting that PI3K-Akt signaling was responsible for the bio-function of these 8 candidate miRNAs. Indeed, PI3K-Akt signaling plays an important role in cancer progression by regulating cell cycle, apoptosis and metastasis. miR-196a was one of these 8 candidate miRNAs.

Previous studies showed that the miR-196a expression was dysregulated in non-small cell lung carcinoma, colorectal cancer, and glioblastoma (31-33). Besides, the high expression of miR-196a promoted esophageal cell proliferation, anchorage-independent growth and suppressed apoptosis by directly targeting Annexin A1 (34). Recent study indicated that miR-196a could enhance gastric cancer cell proliferation by directly binding and downregulating p27 ${ }^{\mathrm{kip} 1}$ (35).

In this study, we found that miR-196a expression was associated with UCA1 in bladder cancer by deep sequencing. Our results demonstrated that the tissue levels of UCA1 and miR-196a were statistically significantly higher in bladder cancer patients than those in non-tumor bladder tissues, whereas p2 $7^{\mathrm{kip} 1}$ expression was remarkably decreased in bladder cancer patients. Similar results were observed in bladder cancer cells when transfecting with the plasmids of pCDNA3.1-UCA1 or shUCA1. Further, UCA1 was positively correlated with miR-196a in bladder cancer pateints, whereas the levels of UCA1 and miR-196a were negatively correlated with $\mathrm{p} 27^{\mathrm{kip} 1}$ in bladder cancer, respectively. p2 $7^{\mathrm{kip} 1}$ was an important downstream molecule of PI3K-Akt signaling. Taken together, the results of deep sequencing demonstrated that miRNAs associated with UCA1 play an important role in bladder cancer progression through PI3K-Akt signaling. Further studies are still needed to reveal the underlying molecular mechanism involved in this signaling pathway.

\section{References}

1. Volders PJ, Helsens K, Wang X, Menten B, Martens L, Gevaert K, Vandesompele J and Mestdagh P: LNCipedia: A database for annotated human IncRNA transcript sequences and structures. Nucleic Acids Res 41: D246-D251, 2013.

2. ENCODE Project Consortium: An integrated encyclopedia of DNA elements in the human genome. Nature 489: 57-74, 2012.

3. Nagano $T$ and Fraser P: No-nonsense functions for long noncoding RNAs. Cell 145: 178-181, 2011.

4. Wapinski $\mathrm{O}$ and Chang HY: Long noncoding RNAs and human disease. Trends Cell Biol 21: 354-361, 2011.

5. Gibb EA, Brown CJ and Lam WL: The functional role of long non-coding RNA in human carcinomas. Mol Cancer 10: 38, 2011.

6. Ponting CP, Oliver PL and Reik W: Evolution and functions of long noncoding RNAs. Cell 136: 629-641, 2009.

7. Wang X, Song X, Glass CK and Rosenfeld MG: The long arm of long noncoding RNAs: Roles as sensors regulating gene transcriptional programs. Cold Spring Harb Perspect Biol 3: a003756, 2011.

8. Bartel DP: MicroRNAs: Target recognition and regulatory functions. Cell 136: 215-233, 2009.

9. Salmena L, Poliseno L, Tay Y, Kats L and Pandolfi PP: A ceRNA hypothesis: The Rosetta Stone of a hidden RNA language? Cell 146: 353-358, 2011.

10. Thomas M, Lieberman J and Lal A: Desperately seeking microRNA targets. Nat Struct Mol Biol 17: 1169-1174, 2010.

11. Djebali S, Davis CA, Merkel A, Dobin A, Lassmann T, Mortazavi A, Tanzer A, Lagarde J, Lin W, Schlesinger F, et al: Landscape of transcription in human cells. Nature 489: 101-108, 2012.

12. Mattick JS: Non-coding RNAs: The architects of eukaryotic complexity. EMBO Rep 2: 986-991, 2001.

13. Mattick JS and Gagen MJ: The evolution of controlled multitasked gene networks: The role of introns and other noncoding RNAs in the development of complex organisms. Mol Biol Evol 18: 1611-1630, 2001.

14. Tay Y, Rinn J and Pandolfi PP: The multilayered complexity of ceRNA crosstalk and competition. Nature 505: 344-352, 2014.

15. Gutschner T and Diederichs S: The hallmarks of cancer: A long non-coding RNA point of view. RNA Biol 9: 703-719, 2012.

16. Kasinski AL and Slack FJ: Epigenetics and genetics. MicroRNAs en route to the clinic: Progress in validating and targeting microRNAs for cancer therapy. Nat Rev Cancer 11: 849-864, 2011.

17. Stahlhut C and Slack FJ: MicroRNAs and the cancer phenotype: Profiling, signatures and clinical implications. Genome Med 5: 111-122, 2013.

18. Lu J, Getz G, Miska EA, Alvarez-Saavedra E, Lamb J, Peck D Sweet-Cordero A, Ebert BL, Mak RH, Ferrando AA, et al: MicroRNA expression profiles classify human cancers. Nature 435: 834-838, 2005.

19. Dvinge H, Git A, Gräf S, Salmon-Divon M, Curtis C, Sottoriva A, Zhao Y, Hirst M, Armisen J, Miska EA, et al: The shaping and functional consequences of the microRNA landscape in breast cancer. Nature 497: 378-382, 2013.

20. Kim TM, Huang W, Park R, Park PJ and Johnson MD: A developmental taxonomy of glioblastoma defined and maintained by MicroRNAs. Cancer Res 71: 3387-3399, 2011.

21. Manterola L, Guruceaga E, Gállego Pérez-Larraya J, GonzálezHuarriz M, Jauregui P, Tejada S, Diez-Valle R, Segura V, Samprón N, Barrena C, et al: A small noncoding RNA signature found in exosomes of GBM patient serum as a diagnostic tool. Neuro-oncol 16: 520-527, 2014. 
22. Tsai MC, Spitale RC and Chang HY: Long intergenic noncoding RNAs: New links in cancer progression. Cancer Res 71: 3-7, 2011.

23. Mercer TR, Dinger ME and Mattick JS: Long non-coding RNAs: Insights into functions. Nat Rev Genet 10: 155-159, 2009.

24. Wang F, Li X, Xie X, Zhao L and Chen W: UCA1, a non-proteincoding RNA up-regulated in bladder carcinoma and embryo, influencing cell growth and promoting invasion. FEBS Lett 582: 1919-1927, 2008

25. Yang C, Li X, Wang Y, Zhao L and Chen W: Long non-coding RNA UCA1 regulated cell cycle distribution via CREB through PI3-K dependent pathway in bladder carcinoma cells. Gene 496: 8-16, 2012.

26. Metzker ML: Sequencing technologies - the next generation. Nat Rev Genet 11: 31-46, 2010.

27. Buermans HP, Ariyurek Y, van Ommen G, den Dunnen JT and 't Hoen PA: New methods for next generation sequencing based microRNA expression profiling. BMC Genomics 11: 716-731, 2010.

28. Anselmo A, Flori L, Jaffrezic F, Rutigliano T, Cecere M, Cortes-Perez N, Lefèvre F, Rogel-Gaillard C and Giuffra E: Co-expression of host and viral microRNAs in porcine dendritic cells infected by the pseudorabies virus. PLoS One 6: e17374 2011.

29. Stark MS, Tyagi S, Nancarrow DJ, Boyle GM, Cook AL, Whiteman DC, Parsons PG, Schmidt C, Sturm RA and Hayward NK: Characterization of the melanoma miRNAome by deep sequencing. PLoS One 5: e9685, 2010.
30. Li M, Liu Y, Wang T, Guan J, Luo Z, Chen H, Wang X, Chen L, Ma J, Mu Z, et al: Repertoire of porcine microRNAs in adult ovary and testis by deep sequencing. Int J Biol Sci 7: 1045-1055, 2011.

31. Wang R, Wang ZX, Yang JS, Pan X, De W and Chen LB: MicroRNA-451 functions as a tumor suppressor in human non-small cell lung cancer by targeting ras-related protein 14 (RAB14). Oncogene 30: 2644-2658, 2011.

32. Guan Y, Mizoguchi M, Yoshimoto K, Hata N, Shono T, Suzuki SO, Araki Y, Kuga D, Nakamizo A, Amano T, et al: MiRNA-196 is upregulated in glioblastoma but not in anaplastic astrocytoma and has prognostic significance. Clin Cancer Res 16: 4289-4297, 2010.

33. Schimanski CC, Frerichs K, Rahman F, Berger M, Lang H, Galle PR, Moehler M and Gockel I: High miR-196a levels promote the oncogenic phenotype of colorectal cancer cells. World J Gastroenterol 15: 2089-2096, 2009.

34. Luthra R, Singh RR, Luthra MG, Li YX, Hannah C, Romans AM, Barkoh BA, Chen SS, Ensor J, Maru DM, et al: MicroRNA-196a targets annexin A1: A microRNA-mediated mechanism of annexin A1 downregulation in cancers. Oncogene 27: 6667-6678, 2008.

35. Sun M, Liu XH, Li JH, Yang JS, Zhang EB, Yin DD, Liu ZL, Zhou J, Ding Y, Li SQ, et al: MiR-196a is upregulated in gastric cancer and promotes cell proliferation by downregulating p27(kip1). Mol Cancer Ther 11: 842-852, 2012. 